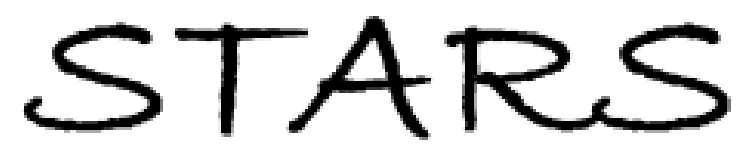

University of Central Florida

STARS

Faculty Bibliography 1990s

Faculty Bibliography

$1-1-1998$

\title{
Spin-glass-like complex susceptibility of frozen magnetic fluids
}

Susamu Taketomi

University of Central Florida

Find similar works at: https://stars.library.ucf.edu/facultybib1990

University of Central Florida Libraries http://library.ucf.edu

This Article is brought to you for free and open access by the Faculty Bibliography at STARS. It has been accepted for inclusion in Faculty Bibliography 1990s by an authorized administrator of STARS. For more information, please contact STARS@ucf.edu.

\section{Recommended Citation}

Taketomi, Susamu, "Spin-glass-like complex susceptibility of frozen magnetic fluids" (1998). Faculty Bibliography 1990s. 2471.

https://stars.library.ucf.edu/facultybib1990/2471

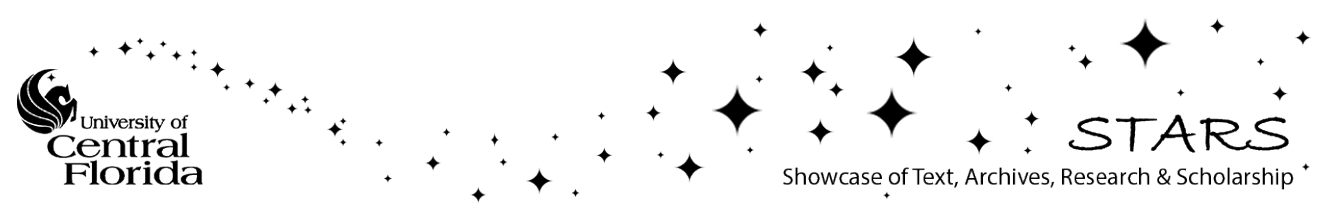




\title{
Spin-glass-like complex susceptibility of frozen magnetic fluids
}

\author{
Susamu Taketomi* \\ Department of Physics, University of Central Florida, Orlando, Florida 32816
}

(Received 5 August 1996)

\begin{abstract}
The complex magnetic susceptibility $\chi=\chi^{\prime}-i \chi^{\prime \prime}$ of different kinds of magnetic fluids (MFs) was measured as a function of temperature $T$ from 6 to $300 \mathrm{~K}$ in a weak ac field of 1 Oe for frequencies ranging from $f=0.1$ to $1000 \mathrm{~Hz}$. A prominent peak appears in both $\chi^{\prime}$ and $\chi^{\prime \prime}$ as a function of $T$ in the frozen state of the MF in which cluster formation of the colloidal particles is difficult, whereas no peak appears in the frozen state of other MFs in which clusters form easily. The peak temperature $T_{p 2}$ of $\chi^{\prime \prime}$ depends on $f$ following the VogelFulcher (VF) law, i.e., $f=f_{0} \exp \left[-E_{s g} / k_{B}\left(T_{p 2}-T_{0}\right)\right]$, where $f_{0}$ and $E_{s g}$ are positive constants and $T_{0}$ is a function of the particles' volume fraction $\phi$. The VF law only holds for $0.0007 \leqslant \phi \leqslant 0.104$, where an empirical power law of $T_{0} \propto \phi^{0.41}$ holds. There is another kind of peak in the loss factor $\tan \delta=\chi^{\prime \prime} / \chi^{\prime}$ as a function of $T$, which means the existence of a magnetic aftereffect. This peak temperature $T_{p 4}$ is far less than $T_{p 2}$ and shown as an Arrhenius-type dependence on $f$ with the exception of a MnZn ferrite particle MF. [S1063-651X(97)10111-8]
\end{abstract}

PACS number(s): 82.70.Dd, 75.50.Mm, 75.40.Gb, 75.50.Lk

\section{INTRODUCTION}

In magnetic fluids (MFs), every colloidal particle consists of a single magnetic domain. The magnetic moment of the domain $\boldsymbol{m}$, behaves as the permanent magnetic dipole of a paramagnetic molecule which has a magnetic moment of a few $\mu_{B}$, whereas the magnetic domain has about $10^{4} \mu_{B}\left(\mu_{B}\right.$ is a Bohr magneton) [1-3]. If a colloidal particle has uniaxial magnetic anisotropy, the direction of $\boldsymbol{m}$ is confined to the direction of the easy axis of the particle, which is also fixed in a frozen MF at low temperatures. With an increase in the temperature $T$ the thermal energy $k_{B} T$ overcomes the barriers of the magnetic anisotropy energy $K v$ enhancing the relaxation of $\boldsymbol{m}$. Here $k_{B}, K$, and $v$ are the Boltzmann constant, magnetic anisotropy constant, and the particle volume, respectively. These rotational relaxations are called Néel relaxations [4]. In addition, when the MF solvent becomes liquid with a further increase in $T$, the colloidal particles begin Brownian rotation, which also causes $\boldsymbol{m}$ to undergo rotational Brownian relaxation. It is believed that an MF is an example of a superparamagnetic material, and many experiments have supported this physical picture [5]. There are, however, a considerable number of studies which throw some doubt on this picture. These experiments include precise magnetization measurements of $\mathrm{MF}$ as a function of temperature [6,7] and magneto-optical experiments on MFs. [8]. Precise magnetization data for the MFs indicate that the Curie-Weiss behavior is obeyed slightly. The magnetic birefringence of magnetic fluids shows generalized Curie-Weiss behavior. Theoretically, Cebers [9], and Sano and Doi [10] discussed the phase separation of the MFs by introducing a mean field induced by the mutual dipole-dipole interaction of the particles. Using the mean spherical model, Morozov

\footnotetext{
*Present address: Dept. of Materials Science and Engineering, University of Washington, Seattle, WA 98195. Permanent address: Matsumoto Yushi-Seiyaku Co. Ltd., Osaka 581, Japan.
}

et al. discussed the magnetization and the magnetic susceptibility of the MFs [11].

The relaxation time of the magnetic dipole of colloidal particles in MFs has been studied in the past [12-15]. Wohlfarth [13], and Chantrell and Wohlfarth [14] pointed out the similarity among metallic alloys diluted with ferrous ions, magnetic rocks, and the MFs, and suggested a VogelFulcher-type relaxation in the MFs.

An ac complex magnetic susceptibility measurement of MF is a suitable method to study the relaxation process of the magnetic dipoles of colloidal particles in MFs. With regard to the liquid MF, Fannin et al. measured ac complex magnetic susceptibility $\chi=\chi^{\prime}-i \chi^{\prime \prime}$ at room temperature [16]. Here $\chi^{\prime}$ and $\chi^{\prime \prime}$ are real and imaginary parts of $\chi$, respectively. In their early papers [16], after Scaife's analysis [15] which is based on Brown's theory of single domain particles [17], Fannin et al. reported that the experimental results were explained by the Debye model [18]. Recently, however, they reported that the results must be interpreted by the magnetic aftereffect [19], which we think must be due to the mutual interaction of the particles. Hanson and Johansson reported that the relation between the peak frequency of $\chi^{\prime \prime}$ and particle concentration suggests that the Vogel-Fulcher law holds [20].

With regard to the frozen MF, Tari et al. measured the ac susceptibility of a MF as a function of temperature and found a peak near $100 \mathrm{~K}$ [21]. Minakov et al. interpreted the change in $\chi$ of the frozen magnetic fluid in terms of some phase transition, something similar to a spin glass transition [22]. Abu-Aljarayesh et al. measured the temperature dependence of $\chi^{\prime}$ of a MF from $80 \mathrm{~K}$ to room temperature and found that the peak temperature of $\chi^{\prime}$ and the ac field frequency qualitatively obeys the Vogel-Fulcher law [23]. Jonsson et al. measured the complex $\chi$ of a $\gamma-\mathrm{Fe}_{2} \mathrm{O}_{3}$ particle $\mathrm{MF}$ and found a prominent magnetic aftereffect [24]. Recently Zhang et al. reported that the peak temperature of the imaginary part $\chi^{\prime \prime}$ obeys the Vogel-Fulcher law and that some scaling relation exists in this law. This suggests that this 
phenomenon is related to some phase transition phenomena [25].

In this paper, we measure the ac complex magnetic susceptibilities of various kinds of MFs as a function of temperature $T$ and frequency $f$ of the ac field. While signals of $\chi^{\prime \prime}$ in the present experiment are weak, we are able to obtain quite precise data using a superconducting quantum interference device (SQUID) susceptometer, the result of which will be discussed from the view point of magnetic aftereffects including disaccommodation and spin-glass phenomena.

\section{EXPERIMENT}

\section{A. Magnetic fluid samples}

The physical properties of the MFs used in the present experiment are tabulated in Table I. All the specimens were provided by Matsumoto Yushi- Seiyaku Co. Ltd. (Marpomagna FV-42, FW-40, FNC-50, and MA-400). The colloidal particles are magnetite and $\mathrm{MnZn}$ ferrite. The solvents are alkylnaphthalenes, water, and paraffin. Though the solvent for sample $\mathrm{D}$ is alkylnaphthalene, the number $n$ of the alkyl group $\left[\mathrm{CH}_{3}\left(\mathrm{CH}_{2}\right)_{n^{-}}\right]$is slightly different from that of the solvent for sample A- $i$ ( $i=1$ to 7$)$. Therefore, we denote the solvent of sample A-i as alkylnaphthalene I and that of sample D as alkylnaphthalene II to distinguish these two different alkylnaphthalenes.

To study the effect of the volume fraction of the colloidal particles on the magnetic susceptibility, several diluted MFs were prepared from the mother $\mathrm{MF}$ of sample A1(Marpomagna FV-42). The magnetic fluid of sample A-6 was prepared by the following method. The Marpomagna FV-42 was placed on a flat glass dish and be held in a ventilator at a temperature of $353 \mathrm{~K}$ for 4 days until the liquid became a gel. By assuming the decrease in weight was totally due to solvent evaporation, we get the volume fraction of the colloidal particles $\phi=0.129$. This is an approximate value because some of the surfactant also evaporated.

In the magneto-optical experiments, we found that MFs which were under vacuum showed different magneto-optical effects compared to MFs which were not held under vacuum [26]. We speculate that vacuum state changed some dispersion state of the colloidal particles in the MF. Therefore, in the present experiment, we prepared a MF of Marpomagna FV-42 which was under a vacuum of $10^{-3}$ torr for $20 \mathrm{~min}$. Using this MF we prepared sample A-7.

The sample is a cylindrical shape of $3 \mathrm{~mm}$ diameter and 6 $\mathrm{mm}$ length. The magnetic and magneto-optical characters of these magnetic fluids have been shown elsewhere [27-29].

\section{B. Experimental procedure}

The ac complex magnetic susceptibility $\chi$ of the MFs was measured by Quantum Design Inc.'s SQUID susceptometer “MPMS2." Each MF sample was first rapidly cooled from room temperature to $4.5 \mathrm{~K}$ with zero field. The cooling rate was approximately $100 \% \mathrm{~min}$. Then the susceptibility $\chi$ was measured at temperature $T$ in intervals of $4^{\circ}$ from 6 to $300 \mathrm{~K}$ for five different frequencies $f=0.1,1,10,100$, and 1000 $\mathrm{Hz}$, respectively.

The ac field amplitude $H_{\text {ac }}$ was 1 Oe except for sample A-5. For sample A-5 the ac amplitude was 5 Oe due to the weak signal. The linear relation between the magnetization and the field was confirmed in this field region.

\section{RESULTS}

The values of $\chi$ depends on the number of colloidal particles per unit volume of the fluid. Therefore dividing $\chi^{\prime}$ and $\chi^{\prime \prime}$ by the volume fraction $\phi$ of the colloidal particles, respectively, we obtain the normalized susceptibilities $\chi^{\prime} / \phi$ and $\chi^{\prime \prime} / \phi$ which are proportional to the susceptibility per particle. Hereafter we call these normalized susceptibilities as merely susceptibilities $\chi^{\prime}$ and $\chi^{\prime \prime}$, respectively. The unit of $\chi$ is the cgs nonrational Gauss unit, G/Oe.

The temperature dependence of $\chi^{\prime}$ and $\chi^{\prime \prime}$ for field frequencies $f=0.1$ and $1000 \mathrm{~Hz}$ for samples A- $i$ ( $i$ $=1,2,3,4,5), B, C$, and D are shown in Figs. 1(a) and 1(b), respectively [30]. Here we denote the temperature of the peak of $\chi^{\prime \prime}$ in the liquid state and that in the frozen state as $T_{p 1}$ and $T_{p 2}$, respectively, after Ref. [25]. The pouring points of MF are shown in Table I.

Samples A-1 through A-5 have large peaks in the frozen state for both $\chi^{\prime}$ and $\chi^{\prime \prime}$, while small or no peaks in the liquid state. On the contrary, sample B has large peaks in the liquid state for both $\chi^{\prime}$ and $\chi^{\prime \prime}$. In the frozen state, there is only a small peak in $\chi^{\prime \prime}$. Samples $C$ and D also show large peaks in $\chi^{\prime \prime}$ in the liquid state while there is no peak in $\chi^{\prime}$ and $\chi^{\prime \prime}$ in the frozen state. (Only a small shoulder in $\chi^{\prime \prime}$ appears in sample D.)

The peak values of $\chi^{\prime}$ and $\chi^{\prime \prime}$ decrease with $\phi$ for samples A-1 through A-5. (Note that $\chi^{\prime}$ and $\chi^{\prime \prime}$ are normalized values with respect to $\phi$.) These dilution effects coincide with those of Jonsson et al. [24]. Figures 2(a)-2(c), show the temperature dependence of $\chi^{\prime}$ and $\chi^{\prime \prime}$ of samples A-1, A-6, and D, respectively, for the five different ac field frequencies $f=0.1,1,10,100$, and $1000 \mathrm{~Hz}$. In Fig. 2(a), the peak value of $\chi^{\prime}$ decreases while that of $\chi^{\prime \prime}$ increases with $f$. Both the peak temperatures of $\chi^{\prime}$ and that of $\chi^{\prime \prime}$ increase with $f$. The peak temperature $T_{p 2}$ of $\chi^{\prime \prime}$ at $f=1000 \mathrm{~Hz}$ is listed in Table I. In addition there are small shoulders in $\chi^{\prime \prime}$ at about $T=30 \mathrm{~K}$. The samples A-2 through A-5 show the same characteristics. On the contrary, in Fig. 2(b) of sample A-6, the shoulder around $T=30 \mathrm{~K}$ in $\chi^{\prime \prime}$ disappears completely while the rest of the characteristics are the same as Fig. 2(a). Here the nominal value $\phi=0.129$ was used for sample A-6. In Fig. 2(c) of sample D, there is no peak in $\chi^{\prime \prime}$ in the frozen state while a prominent peak exists in the liquid state. The peak values of both $\chi^{\prime}$ and $\chi^{\prime \prime}$ in the liquid state decreases with $f$, while the peak temperatures of $\chi^{\prime}$ and $\chi^{\prime \prime}$ increase with $f$. The peak temperature $T_{p 1}$ of $\chi^{\prime \prime}$ at $f=1000 \mathrm{~Hz}$ is listed in Table I.

\section{DISCUSSION}

Many authors who studied the complex magnetic susceptibility of frozen MFs, took for granted that the peak in $\chi^{\prime \prime}$ as a function of the temperature is due to the resonant effect of Néel relaxation of the dipoles. In this paper, however, we discuss the same phenomenon from a different viewpoint, i.e., the view of phenomenological magnetic aftereffect. 


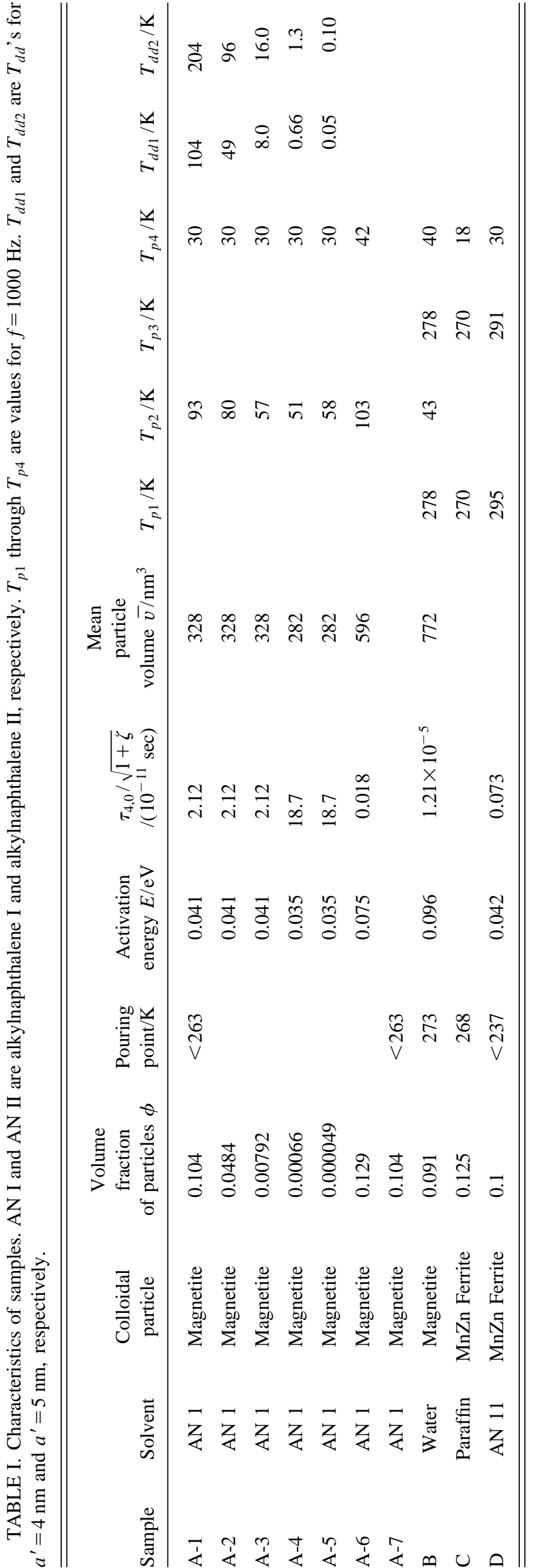

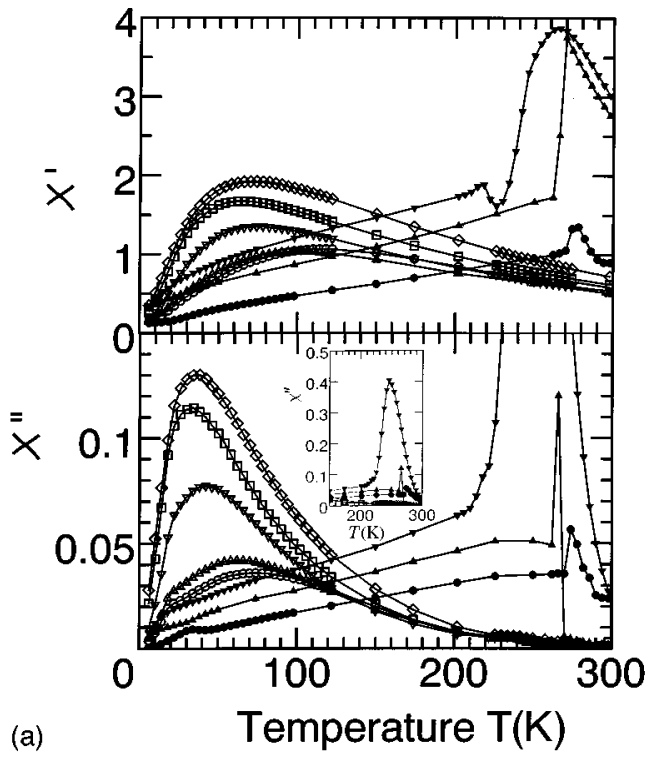

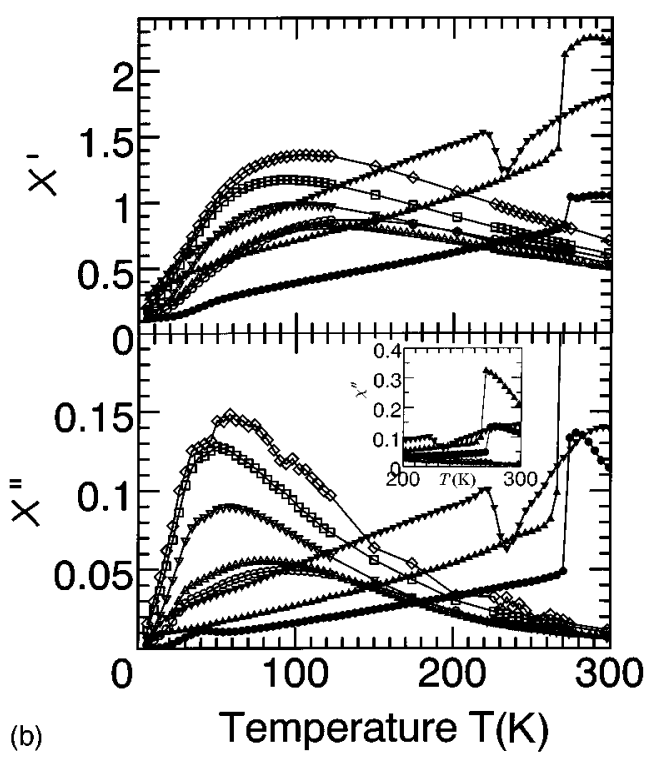

FIG. 1. $\chi^{\prime}$ and $\chi^{\prime \prime}$ as a function of temperature $T$ for an ac field $f$ of 0.1 and $1000 \mathrm{~Hz}$. (a) $f=0.1 \mathrm{~Hz}$, (b) $f=1000 \mathrm{~Hz}, \bigcirc: \mathrm{A}-1, \triangle$ : A-2, $\nabla:$ A-3, $\square:$ A-4, $\diamond:$ A-5, ○: B, $\mathbf{\Delta}:$ C, $\nabla:$ D.

\section{A. Magnetic aftereffect}

\section{Phenomenological approach}

Generally speaking, if magnetic material has a magnetic aftereffect, the time dependence of the magnetization $M$ is phenomenologically expressed by [31]

$$
\frac{d\left(M-\chi_{S} H\right)}{d t}=-\frac{1}{\tau_{4}}\left[M-\chi_{S} H(1+\zeta)\right],
$$

where $H$ is an external magnetic field, $\chi_{S}, \tau_{4}$, and $\zeta$ are positive constants corresponding to the so-called adiabatic or instantaneous susceptibility, relaxation time constant, and ratio of the change in magnetization by the after effect over the initial magnetization, respectively. The meaning of the subscript 4 in $\tau_{4}$ will be clarified later.

When an ac external magnetic field $H=H_{0} e^{i \omega t}$, is applied to the material, the magnetization $M$ has a phase lag 

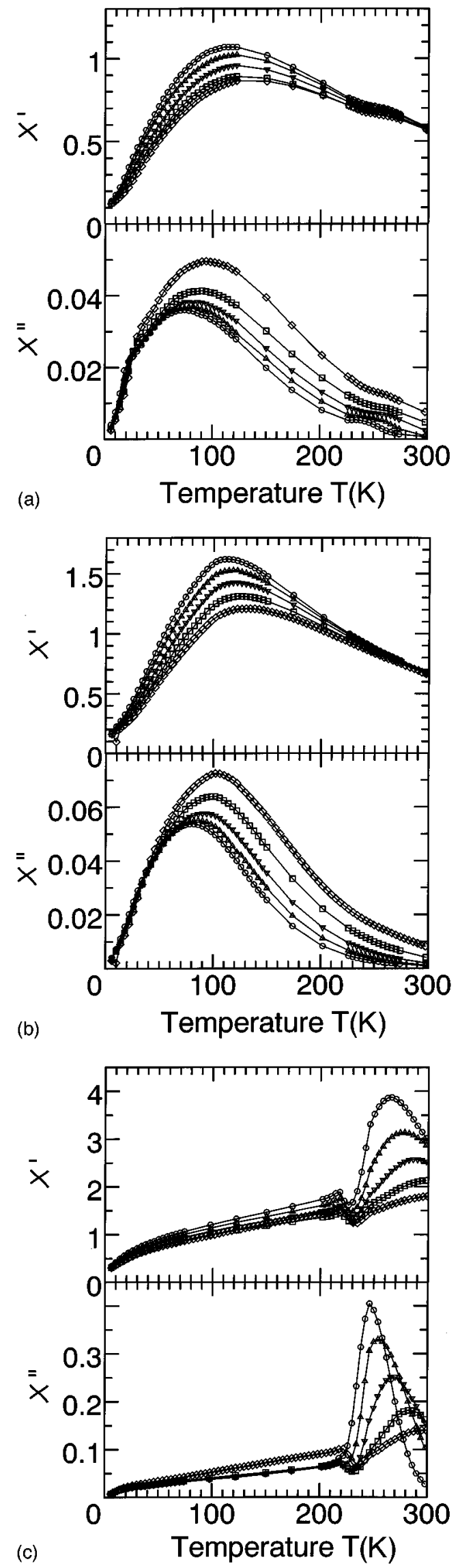

FIG. 2. $\chi^{\prime}$ and $\chi^{\prime \prime}$ of samples A-1, A-6, and D as a function of temperature $T$ for ac field frequencies $f$. (a) A-1, (b) A-6, (c) D, $\bigcirc$ : $0.1 \mathrm{~Hz}, \triangle: 1 \mathrm{~Hz}, \nabla: 10 \mathrm{~Hz}, \square: 100 \mathrm{~Hz}, \diamond: 1000 \mathrm{~Hz}$.
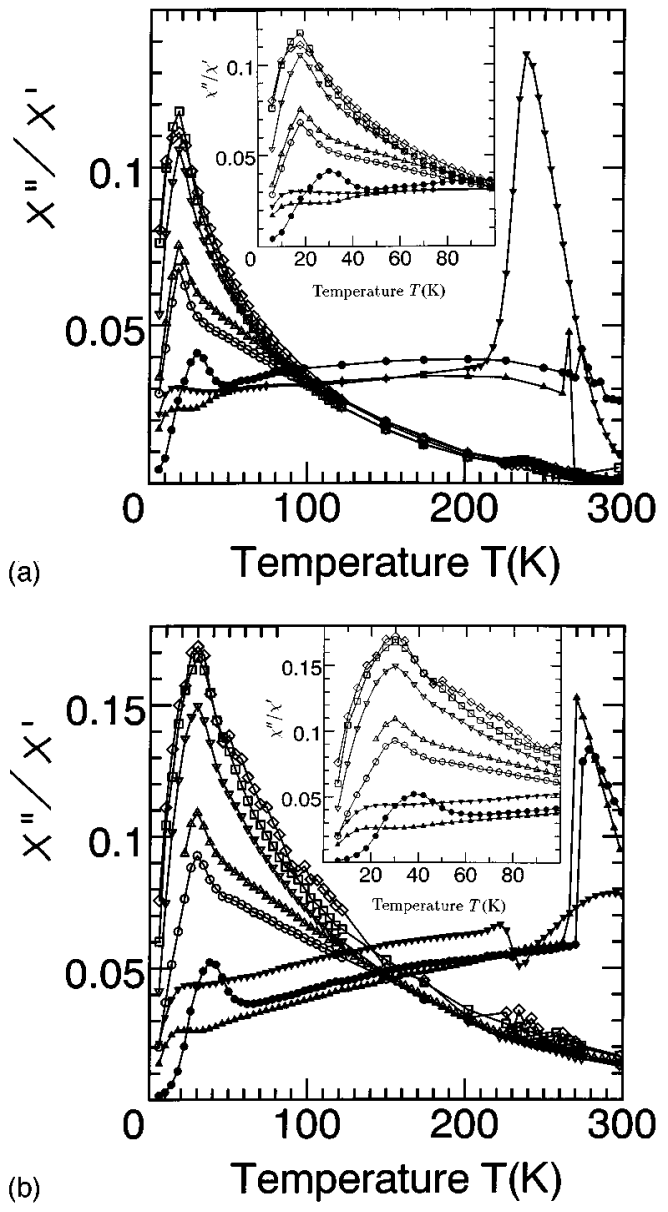

FIG. 3. $\chi^{\prime \prime} / \chi^{\prime}$ as a function of temperature for an ac field $f$ of 0.1 and $1000 \mathrm{~Hz}$. (a) $f=0.1 \mathrm{~Hz}$, (b) $f=1000 \mathrm{~Hz}, \bigcirc: \mathrm{A}-1, \triangle$ : A-2, $\nabla:$ A-3, $\square:$ A-4, $\diamond:$ A-5, $\mathbf{0}:$ B, $\mathbf{\Delta}:$ C, $\nabla:$ D.

$M=M_{0} e^{i \omega t-\delta}$, where $\omega$ is an angular frequency of the external ac field, $H_{0}$ and $M_{0}$ are amplitudes, and $\delta$ is the socalled loss angle. $\delta$ is expressed by [31]

$$
\begin{aligned}
\tan \delta & =\frac{\zeta \omega \tau_{4}}{(1+\zeta)+\left(\omega \tau_{4}\right)^{2}} \\
& =\frac{\chi^{\prime \prime}}{\chi^{\prime}} .
\end{aligned}
$$

We plot $\chi^{\prime \prime} / \chi^{\prime}$ vs $T$ in Figs. 3(a), 3(b) for samples A- $i$ $(i=1,2,3,4,5), \mathrm{B}, \mathrm{C}$, and $\mathrm{D}$ at $f=0.1$ and $1000 \mathrm{~Hz}$, respectively. Let us denote the peak temperature of $\chi^{\prime \prime} / \chi^{\prime}$ peak in the liquid state and the frozen state as $T_{p 3}$ and $T_{p 4}$, respectively. The peak at $T_{p 4}$ correspond to the shoulders of $\chi^{\prime \prime}-T$ curves in Fig. 2(a). The main peaks in the frozen state in Figs. 1, on the contrary, disappear in Fig. 3. The peak temperature $T_{p 4}$ in $\chi^{\prime \prime} / \chi^{\prime}$ is independent of the colloidal volume fraction $\phi$ with $\phi \leqslant 0.104$, while those of samples A-6, B, and D differ from one another. The values of $T_{p 3}$ and $T_{p 4}$ at $f=1000 \mathrm{~Hz}$ are listed in Table $\mathrm{I}$.

Figures 4(a), 4(b) show $\chi^{\prime \prime} / \chi^{\prime}$ vs $T$ curves of samples A-1 and A-6, respectively, for five different values of $f$. Both the $\chi^{\prime \prime} / \chi^{\prime}$ peak value and $T_{p 4}$ increases with increase of $f$ for both samples. There is, however, a bend in slope in the former curves after passing the peak while there is not for the 


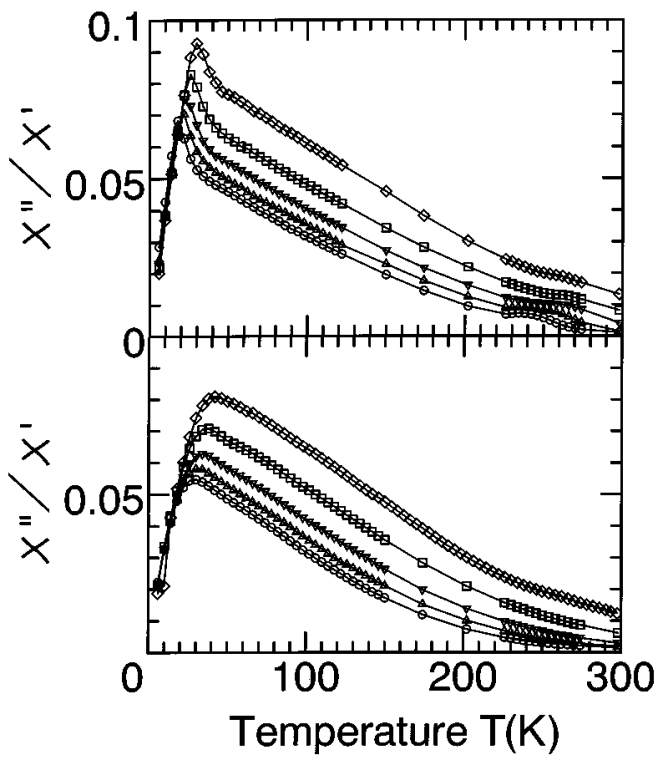

FIG. 4. $\chi^{\prime} / \chi^{\prime}$ of samples A-1 and A-6 as a function of temperature for ac field frequencies $f$. (a) upper: A-1, (b) lower: A-6, $\bigcirc$ : $0.1 \mathrm{~Hz}, \triangle: 1 \mathrm{~Hz}, \nabla: 10 \mathrm{~Hz}, \square: 100 \mathrm{~Hz}, \diamond: 1000 \mathrm{~Hz}$.

latter. Figure 5 shows the $T_{p 4}$ vs $\log f$ for sample A-1. It shows that the relaxation time $\tau_{4}$ expressed by

$$
\tau_{4}=\frac{\sqrt{1+\zeta}}{2 \pi f}
$$

precisely obeys the Arrhenius law

$$
\tau_{4}=\tau_{4,0} \exp \left[\frac{E}{k_{B} T}\right]
$$

where $\tau_{4,0}$ is a constant and $E$ is an activation energy. The $T_{p 4}$ vs $\log f$ relation of all other samples also satisfy Arrhenius law. The values of $\tau_{4,0} / \sqrt{1+\zeta}$ and $E$ obtained from the experimental data of $T_{p 4} \mathrm{vs} \log f$ straight lines are shown in Table I.

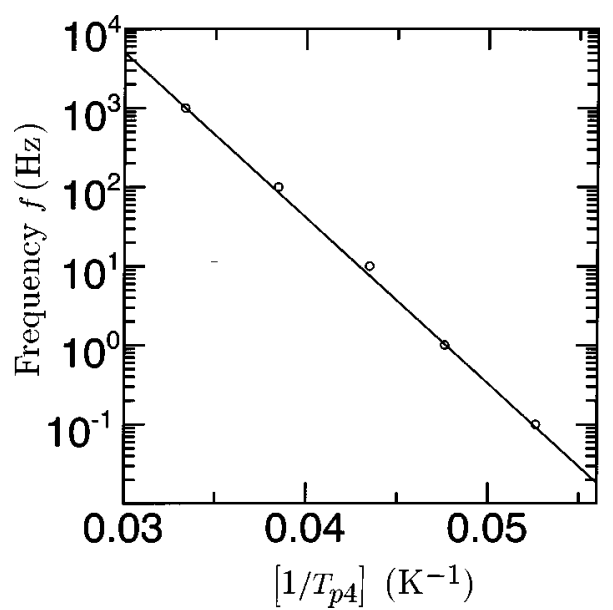

FIG. 5. Arrhenius plot of the peak temperature $T_{p 4}$ vs the frequency $f$ for sample A-1.

\section{Two-state model of dipoles in frozen MF}

For zero field cool frozen MFs, the easy axes of the magnetic moment of the colloidal particles are oriented randomly in direction. When a weak external field $H$ is applied to this frozen MF, Eq. (1) is derived microscopically, if we assume the following two-state model. The two-state model assumes that the magnetic dipole in the colloidal particle of the frozen MF orients almost in the two opposite directions of energy minimum states near the easy axis direction, and that the dipole changes directions by thermal fluctuation going over the energy barrier. (In Appendix A, it is clarified that the dipole does not need to rotate and go over the barrier. Other type of transition of the dipole such as electron hopping is possible if the potential satisfies conditions.) The derivation is shown in Appendix A. From this derivation, the following relations are obtained:

$$
\tau_{4}=\tau_{4,0} \exp \left[\frac{K v}{k_{B} T}\right]
$$

where $K$ and $v$ are magnetic anisotropy constant and volume of the colloidal particle, respectively, $\tau_{4,0}$ is given by

$$
\tau_{4,0}=\frac{1}{4 c}
$$

where $c$ is the rate coefficient of the rate Eq. (A16) in Appendix A. $\chi_{S}, \zeta$ are also derived in Appendix A as

$$
\chi_{S}=\frac{M_{S}^{2} \phi}{3 K},
$$

where $M_{S}$ is the saturation magnetization of the colloidal particles.

$$
\zeta=\frac{\chi_{T}}{\chi_{S}}
$$

where $\chi_{T}$ is the static or isothermal susceptibility expressed by

$$
\chi_{T}=\frac{M_{S}^{2} \phi^{2}}{3 N k_{B} T} .
$$

See Eqs. (A22), (A30), and (A31) in Appendix A.

The anisotropy constant $K$ is due to both the magnetocrystalline anisotropy and the shape anisotropy of the particles. If we assume $K=2 \times 10^{5} \mathrm{erg} / \mathrm{cc}$ which is the magnetocrystalline anisotropy constant of bulk magnetite in the vicinity of $T=30 \mathrm{~K}$ [32], the mean particle volume $\bar{v}$ can be obtained from $E$ and is listed in Table I.

It is well known that the distribution function $f_{\text {dis }}(v)$ of the colloidal particles of volume $v$ in the MF is a log-normal function expressed by [33]

$$
f_{\text {dis }}(v)=\frac{1}{\sqrt{2 \pi} \sigma v} \exp \left[\frac{-\left[\ln \left(v / v_{0}\right)\right]^{2}}{2 \sigma^{2}}\right],
$$




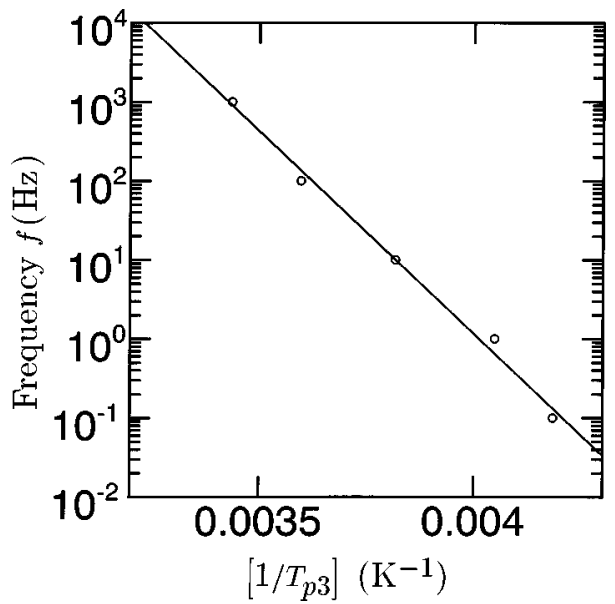

FIG. 6. Arrhenius plot of the peak temperature $T_{p 3}$ vs the frequency $f$ for sample D.

where $v_{0}$ and $\sigma$ are positive constants. The values of $v_{0}=157 \mathrm{~nm}^{3}$ and $\sigma=1.35$ for the MF used for sample A-1 were already obtained by magnetization curve measurement [34]. Therefore the mean volume $\bar{v}$ of the colloidal particles are given by

$$
\begin{aligned}
\bar{v} & =\int_{0}^{\infty} v f_{\text {dis }}(v) d v \\
& =v_{0} \exp \left[\frac{\sigma^{2}}{2}\right] \\
& =391 \mathrm{~nm}^{3} .
\end{aligned}
$$

(See Appendix B.) The agreement of this value and the values of the volume obtained in Table I is fairly well if we take the ambiguity of $K$ into account. The disagreement of $\bar{v}$ of sample A-6 from those of other samples A- $i$ 's is attributed to the growth of the particles during preparation heat treatment. The difference of $v$ of sample $\mathrm{B}$ in Table $\mathrm{I}$ is also attributed to the difference of the colloidal size distribution due to different fabrications condition of the particles. But as is to be clarified in the following section, the physical picture should be once again examined in Sec. IV C.

Using the same value of $K=2 \times 10^{5} \mathrm{erg} / \mathrm{cc}$ and Eq. (7), the normalized adiabatic susceptibility $\bar{\chi}_{S} \equiv \chi_{S} / \phi$ is estimated to be 0.43 , where $M_{s}=509 \mathrm{G}$ at $T=0$ is used. Comparing this value with $\chi^{\prime}$ in Figs. 1 and $2, \bar{\chi}_{S}$ is larger than the real value. This leads to the real $K$ value being much larger, and $K$ might not be due to the magnetocrystalline anisotropy of the particles. We also discuss it again in Sec. IV C.

\section{Liquid state}

Figure 6 shows the $1 / T_{p 3}$ vs $\log f$ relation of sample D in the melted state. It shows the same Arrhenius law of Eq. (4) holds for this peak. The physical mechanism, however, is completely different. In this case, the particle itself can rotate in the solvent and particles are subjected to so-called rotational Brownian relaxation. The relaxation time constant, $\tau_{B}$, of the rotational Brownian relaxation is expressed by [35]

$$
\tau_{B}=\frac{3 v \eta}{k_{B} T},
$$

where $\eta$ is a viscosity of the solvent. As $\eta$ obeys the Arrhenius law [36]

$$
\eta=\eta_{0} \exp \left[\frac{E_{\mathrm{vis}}}{k_{B} T}\right],
$$

$\tau_{B}$ approximately obeys the Arrhenius law

$$
\tau_{B}=\frac{3 v \eta_{0}}{k_{B} T} \exp \left[\frac{E_{\mathrm{vis}}}{k T}\right],
$$

where $E_{\text {vis }}$ is an activation energy for the viscosity of the solvent. Unfortunately we do not have the data on $E_{\text {vis }}$ of alkylnaphthalene II of sample D. The value of $E_{\text {vis }}$ of alkylnaphthalene I which is almost similar to alkylnaphthalene II is known to be $0.516 \mathrm{eV}$ [36]. The value of $E_{\text {vis }}$ obtained from $1 / T_{p 3}$ vs $\log f$ line is $1.016 \mathrm{eV}$. The agreement of these two $E_{\text {vis }}$ values are good in order of magnitude. Therefore it is concluded that this peak is assigned to the rotational Brownian relaxation of the particles.

\section{B. Spin-glass-like behavior}

In this subsection, we discuss the temperature dependence of $\chi^{\prime \prime}$ in frozen MFs, the dipole-dipole interaction of colloidal particles and spin-glass-like behavior of the frozen MF.

\section{Contradiction of neglect of dipole-dipole interaction}

We adopt the following Debye-type formula of the susceptibility $\chi(\omega, T)$ as functions of external ac magnetic field of the angular frequency $\omega$ and the temperature $T$ after Lundgren et al. [37] expressed by

$$
\begin{aligned}
& \chi^{\prime}(\omega, T)=\bar{\chi}_{S}+\int_{\tau_{\min }}^{\tau_{\max }} \frac{\left[\bar{\chi}_{T}-\bar{\chi}_{S}\right] g\left(\tau_{2}\right)}{1+\left(\omega \tau_{2}\right)^{2}} d\left(\ln \tau_{2}\right), \\
& \chi^{\prime \prime}(\omega, T)=\int_{\tau_{\min }}^{\tau_{\max }} \frac{\left[\bar{\chi}_{T}-\bar{\chi}_{S}\right] \omega \tau_{2} g\left(\tau_{2}\right)}{1+\left(\omega \tau_{2}\right)^{2}} d\left(\ln \tau_{2}\right),
\end{aligned}
$$

where $\bar{\chi}_{T}$ and $\bar{\chi}_{S}$ are normalized isothermal and adiabatic susceptibilities with respect to $\phi$, respectively, and $g\left(\tau_{2}\right)$ is a distribution function of the relaxation time constant $\tau_{2} \cdot \tau_{\text {min }}$ and $\tau_{\max }$ are the lower and upper limits of the integral variable $\tau_{2}$. (Debye-type formulas are derived through linear approximation of a relaxation equation. Therefore if the dipole-dipole interaction effect of the colloidal particles are included in the nonlinear term of the relaxation equation, adoption of Deby formula itself means an implicit approximation of neglect of the dipole-dipole interaction.)

Now, in the following we take the non-dipole-dipole interaction approximation and derive the contradiction with the experimental results. We assume that the relaxation time constant $\tau_{2}$ obeys Néel relaxation expressed by

$$
\tau_{2}=\tau_{0} \exp [\beta K v]
$$


where $\tau_{0}$ is a constant, $\beta \equiv 1 /\left(k_{B} T\right)$. This assumption is equivalent to neglecting dipole-dipole interactions. In addition, we assume that $\bar{\chi}_{T}$ is expressed, as usual, using the initial susceptibility formula of paramagnetic materials, by [38]

$$
\begin{aligned}
\bar{\chi}_{T} & =\frac{N\left(M_{S} v\right)^{2}}{3 k_{B} T \phi} \\
& =\frac{M_{S}^{2} v^{2}}{3 k_{B} T \bar{v}},
\end{aligned}
$$

where the expression of $\bar{v}$ is already obtained in Eq. (11). Here we used the facts that the log-normal distribution of the colloidal particles expressed by Eq. (10) and $\bar{\chi}_{S}$ is negligibly small compared with $\bar{\chi}_{T}$. As a matter of fact, in our experiment, $\chi^{\prime}$ seems to converge to 0 with $f$ and it is speculated the above assumption is valid. by

From these assumptions, Eqs. (15) and (16) are expressed

$$
\begin{aligned}
& \chi^{\prime}(\omega, T)=\frac{M_{S}^{2}}{3 k_{B} T \bar{v}} \int_{0}^{\infty} \frac{1}{1+\left(\omega \tau_{0} \exp [\beta K v]\right)^{2}} v^{2} f_{\mathrm{dis}}(v) d v, \\
& \chi^{\prime \prime}(\omega, T)=\frac{M_{S}^{2}}{3 k_{B} T \bar{v}} \int_{0}^{\infty} \frac{\omega \tau_{0} \exp [\beta K v]}{1+\left(\omega \tau_{0} \exp [\beta K v]\right)^{2}} v^{2} f_{\mathrm{dis}}(v) d v .
\end{aligned}
$$

Ordinarily, the upper limit of the integral variable $v^{*}$ is given by

$$
\frac{K v^{*}}{k_{B} T}=1
$$

In this case the integrands themselves converge to zero, rapidly with $v$. We spread formally the upper limit of integration $v$ to infinity.

Now we will show that Eqs. (19) and (20) contradict the experimental results. The first contradiction is a dilution effect. Samples A-2 through A-5 are the diluted MFs of sample

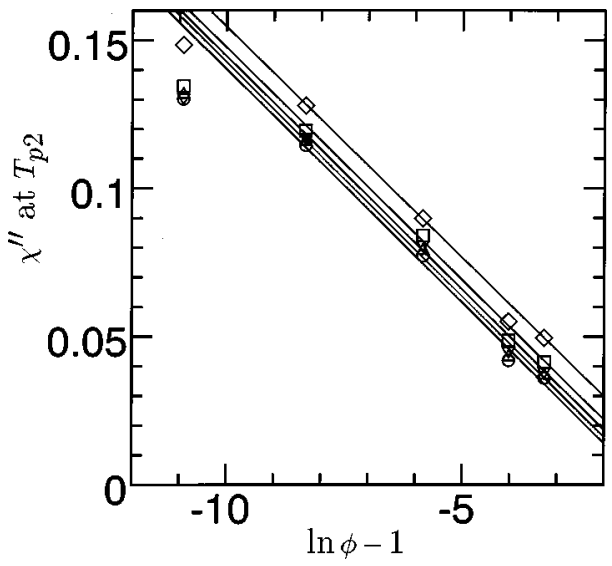

FIG. 7. Peak value of $\chi^{\prime \prime}$ at $T=T_{p 2}$ vs $(\ln \phi-1)$ relation. $\bigcirc: 0.1$ $\mathrm{Hz}, \triangle: 1 \mathrm{~Hz}, \nabla: 10 \mathrm{~Hz}, \square: 100 \mathrm{~Hz}, \diamond: 1000 \mathrm{~Hz}$. The samples are A-1, A-2, A-3, A-4, and A-5.

A-1. Therefore neither $f_{\text {dis }}(v)$ nor $\tau_{2}(T)$ are influenced by the dilution and neither the peak value of $\chi^{\prime \prime}$ nor should $T_{p 2}$ be influenced by the dilution from Eq. (20) for samples A-2 through A-5. The experimental results show, on the contrary, that not only the peak temperature $T_{p 2}$ but also the peak values of $\chi^{\prime \prime}$ change from one sample to another. Figure 7 shows the peak values of $\chi^{\prime \prime}$ as a function of

$$
\ln \phi-1 \text {. }
$$

The experimental data falls approximately on a straight lines except for the lowest concentration sample. As $\chi^{\prime \prime}$ is the normalized $\chi^{\prime \prime}$, the real $\chi^{\prime \prime}$ is proportional to

$$
\phi \ln \phi-\phi \text {. }
$$

Therefore the peak value of $\chi^{\prime \prime}$ is approximately proportional to the mixing entropy

$$
S_{\text {mix }}=k(\phi \ln \phi-\phi) .
$$

This means the configuration of all the particles in the solvent is closely connected to the $\chi^{\prime \prime}$ value.

The second contradiction is with regard to the ratio of $\chi^{\prime \prime} / \chi^{\prime}$ expressed by

$$
\frac{\chi^{\prime \prime}(\omega, T)}{\chi^{\prime}(\omega, T)}=\left[\int_{0}^{\infty} \frac{\omega \tau_{0} \exp [\beta K v]}{1+\left(\omega \tau_{0} \exp [\beta K v]\right)^{2}} v^{2} f_{\mathrm{dis}}(v) d v\right] /\left[\int_{0}^{\infty} \frac{1}{1+\left(\omega \tau_{0} \exp [\beta K v]\right)^{2}} v^{2} f_{\mathrm{dis}}(v) d v\right]
$$

The experimental results show that both of the peaks of $\chi^{\prime}$ and $\chi^{\prime \prime}$ disappear in $\chi^{\prime \prime} / \chi^{\prime}$ curve, which cannot be explained by Eq. (23).

The third contradiction is with respect to the function

$$
R\left(\omega \tau_{2}\right) \equiv \frac{\omega \tau_{2}}{1+\left(\omega \tau_{2}\right)^{2}}
$$

The function $R\left(\omega \tau_{2}\right)$ is an increasing function in the region $\omega \tau_{2}<1$ and after passing the maximum at $\omega \tau_{2}=1$, it becomes a decreasing function in the region $1<\omega \tau_{2}$. If we fix the temperature $T$ far less than $T_{p 2}$ for $\omega=2 \pi \times 0.1$, then $\chi^{\prime \prime}(\omega, T)$ should be a decreasing function with respect to $\omega$ in the region $2 \pi \times 0.1<\omega<2 \pi \times 1000$ because

$$
2 \pi \times 0.1 \tau_{2}\left(T_{p 2}\right)<\omega \tau_{2}\left(T_{p 2}\right)<\omega \tau_{2}(T) .
$$

If we fix the temperature $T$ much higher than $T_{p 2}$ for $\omega=2 \pi \times 1000$, then $\chi^{\prime \prime}(\omega, T)$ should be an increasing function with respect to $\omega$ in the region $2 \pi \times 0.1<\omega<2 \pi \times 1000$ because

$$
\omega \tau_{2}(T)<\omega \tau_{2}\left(T_{p 2}\right)<2 \pi \times 1000 \tau_{2}\left(T_{p 2}\right) .
$$




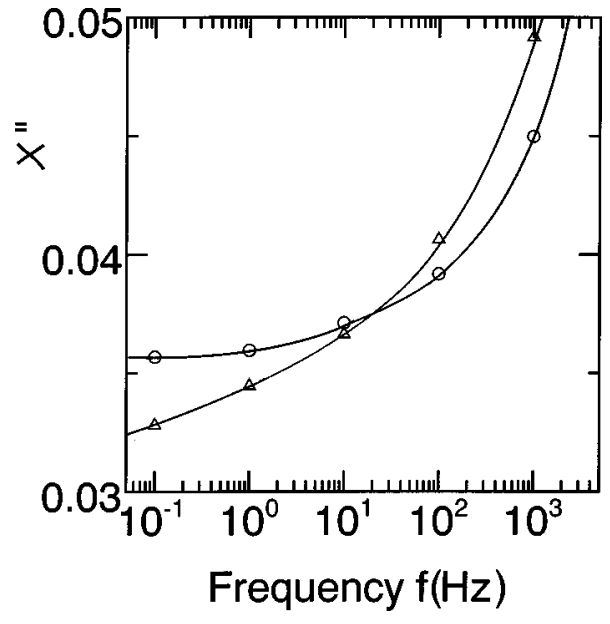

FIG. 8. $\chi^{\prime \prime}$ dependence on $f$ for sample A-1 at fixed temperature T. $\bigcirc: T=66 \mathrm{~K}, \triangle: T=102 \mathrm{~K}$.

Figure 8 shows experimental results of sample A-1. In this case $T_{p 2}$ of $\omega=2 \pi \times 0.1$ is $74 \mathrm{~K}$, while $T_{p 2}$ of $\omega=2 \pi \times 1000$ is $93 \mathrm{~K}$. Therefore we take two temperatures $66 \mathrm{~K}$ and $102 \mathrm{~K}$. Both curves of $T=66 \mathrm{~K}$ and $T=102 \mathrm{~K}$ in Fig. 10 are increasing functions with respect to $\omega$, which contradict the previous prediction.

In conclusion even if we take the relaxation time distribution into account, the experimental results of $T$ dependence on $\chi$ does not agree with the formula of $\chi$ without the dipoledipole interaction. Therefore the dipole-dipole interaction of the colloidal particles is essential for the magnetic susceptibility of the frozen MF.

\section{Estimation of dipole-dipole interaction}

In this subsection it is shown that if the particles are dispersed uniformly in the MF, the calculated dipole-dipole interaction energy is negligible and contradicts with Sec. IV B 1. Let us estimate a dipole-dipole interaction energy $E_{d d}$ between two particles. For convenience, let us assume that all the particles are the sphere of the same radius $a$ and are arranged in cubic lattice of length $l$. Ignoring the sign, $E_{d d}$ of the neighboring two particles whose dipoles are aligned in the same direction are expressed by

$$
E_{d d}=\frac{2 \times\left(4 \pi M_{S} a^{3} / 3\right)^{2}}{l^{3}} .
$$

The lattice length $l$ is expressed with respect to the volume fraction $\phi$ of the colloidal particles by

$$
l^{3}=\frac{4 \pi a^{3}}{3 \phi},
$$

Eq. (25) is transformed to

$$
E_{d d}=\frac{1}{6 \pi}\left(4 \pi M_{S}\right)^{2} a^{3} \phi
$$

For magnetite, as $4 \pi M_{S}=6400 \mathrm{G}$, the ratio of $E_{d d} / k_{B} T$ is expressed by

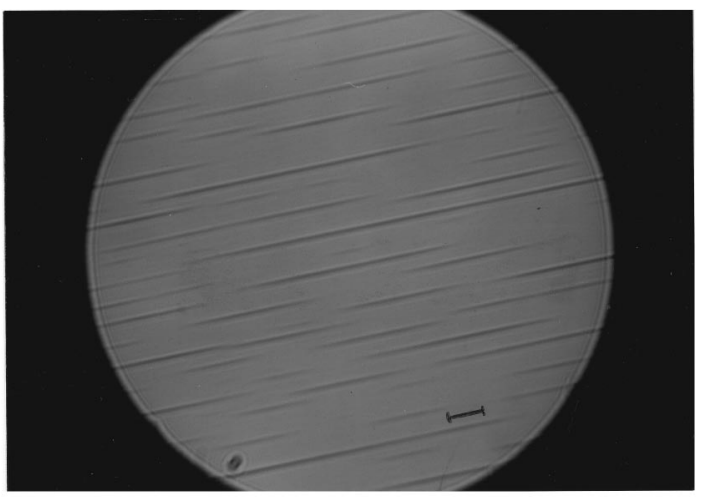

FIG. 9. Optical micrograph of clusters in MF. The external field $H=270$ Oe is applied to tangential direction and $T=295 \mathrm{~K}$. The bar in the graph is $10 \mu \mathrm{m}$. MF is the MF of sample C.

$$
\frac{E_{d d}}{k_{B} T}=15.7 \times \frac{a^{\prime 3} \phi}{T},
$$

where $a^{\prime}$ is the same as $a$ but is scaled in units of nm.

Let us define a threshold dipole-dipole interaction temperature $T_{d d}$ as

$$
\frac{E_{d d}}{k_{B} T_{d d}} \equiv 1
$$

The values of $T_{d d}, T_{d d 1}$, and $T_{d d 2}$ for $a^{\prime}=4 \mathrm{~nm}$ and $a^{\prime}=5 \mathrm{~nm}$, respectively, are tabulated in Table I. The values of $T_{d d}$ for samples A-3, A-4, and A-5 are so low that the particles for these samples should behave with non-dipoledipole interaction in the temperature range of the present experiment. The experimental results are contrary. Therefore the uniform dispersion assumption should be rejected.

\section{Cluster formation or phase separation}

In Sec. IV B 2 we rejected the uniform dispersion assumption of the particles from the evaluation of the dipoledipole interaction energy. The rejection of the uniform dispersion was also confirmed by direct optical microscope observation of MFs [39,40]. Figure 9 is the optical micrograph of the MF (MF of sample $\mathrm{C}$ ) in the presence of an external field of $270 \mathrm{Oe}$ at $T=295 \mathrm{~K}$ [39]. Needlelike clusters or particle-dense phase appears with the external field. The clusters also appear in the MF of sample B while no clusters were observed for the MF of sample A-1 [39]. (This does not mean the phase separation did not occur in the MF of the sample A-1. Since this was the optical microscope observation, the generated clusters might be less than micron dimension or the difference of concentration between the two phases was so small that the clusters were not identified in the micrograph.) The cluster generation was also observed with decreasing temperature [41].

The cluster generation means the phase separation of the $\mathrm{MF}$; the clusters are the dense phase and the rest of the region is the diluted phase $[9,10]$. The number density of the colloidal particles in dense phase or the clusters increases 
dramatically while that of the diluted phase decreases dramatically when compared with before phase separation. Therefore the dipole-dipole interaction in the dense phase or the clusters also increases dramatically compared with prephase separation. Even in the dense phase, the dipoledipole interaction strength differs from sample to sample because the number density of the particles in the dense phase is a function of the initial particles concentration, temperature, applied field, and dispersing ability of the surfactant of every sample.

It is naturally speculated that a strong enough interaction causes the ferromagnetic like state, i.e., the dipoles of all the particles in one cluster orient in the same direction. In this case the relaxation time in the frozen state is so long that there is no peak in $\chi^{\prime \prime}-T$ curves in the present experimental temperature and frequency region. The experimental results of the samples B, C, and D correspond to this case. On the other hand, if the interaction of the particles in the cluster is intermediate strength, the relaxation behavior is neither Néel relaxation nor the ferromagneticlike one. The experimental results of the samples A- $i(i=1,2,3,4,5,6)$ correspond to this case. We will discuss it in Sec. IV B 4.

Even in the liquid state, when a transverse magnetic field $H_{\perp}$ is applied to MF, $\chi$ changes greatly as a function of $H_{\perp}$ [42]. This phenomenon was discussed theoretically with regard to cluster formation [43]. Here the direction of $H_{\perp}$ is perpendicular to the ac measuring field of $\chi$.

\section{Vogel-Fulcher law}

It is well known that if the interaction of the magnetic dipoles of atoms are not strong enough to create a ferromagnetic state or antiferromagnetic state, but strong enough compared with that of paramagnetic atoms, the material shows a spin-glass state. Some metallic alloys diluted with ferrous ions show a typical spin-glass state. This susceptibility $\chi$ shows a cusp as a function of temperature and the peak temperature obeys the Vogel-Fulcher law [44].

Zhang et al. proposed that the peak temperature $T_{p 2}$ of $\chi^{\prime \prime}$ in the frozen state is connected to the relaxation time constant $\tau_{2}$ with Vogel-Fulcher law [25]

$$
\tau_{2}=\tau_{2,0} \exp \left[\frac{E_{s g}}{k_{B}\left(T_{p 2}-T_{0}\right)}\right],
$$

where $\tau_{2,0}, E_{s g}$, and $T_{0}$ are positive constants.

Some objections might be raised about this peak. Physical properties of magnetite change greatly in the vicinity of the Verwey temperature of about $120 \mathrm{~K}$ [31]. That is due to the $\chi^{\prime \prime}$ peak. In fact, the magnetocrystalline anisotropy constant $K$ changes in value around the Verwey temperature [31]. The $\gamma-\mathrm{Fe}_{2} \mathrm{O}_{3}$ particle MF, however, shows the same kind of $\chi^{\prime \prime}$ peak in the range of 20 to $40 \mathrm{~K}$ [24], and recently Mamiya and Nakatani reported that FeN particle MFs also shows the $\chi^{\prime \prime}$ peak in the same temperature range as that of magnetite MFs [45]. Therefore the $\chi^{\prime \prime}$ peak in the frozen state of the MF is the characteristic feature of the MF irrespective of particles' material characteristics.

As $\tau_{2}=1 / 2 \pi f$, Eq. (30) is transformed to

$$
T_{p 2}-T_{0}=\frac{E_{s g}}{k_{B}} \frac{1}{\ln f_{0}-\ln f},
$$

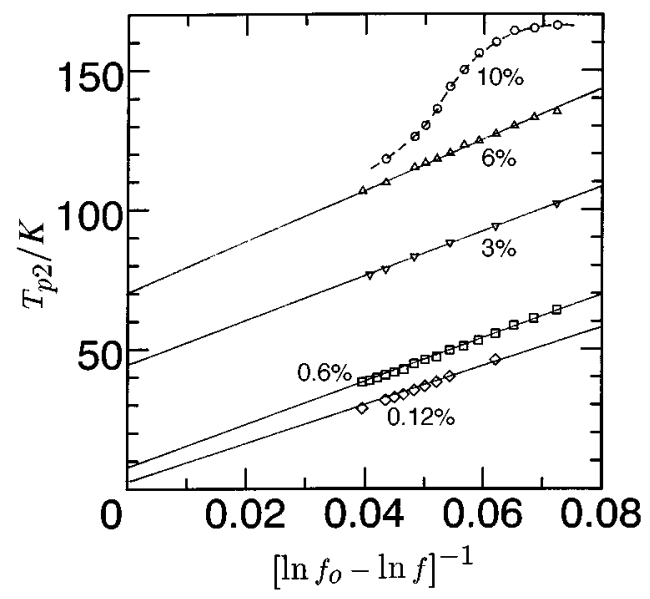

FIG. 10. $T_{p 2}$ vs $\left(\ln f_{0}-\ln f\right)^{-1}$ relations. $\left(\ln f_{0}-\ln f\right)^{-1}$ is scaled in the abscissa, while $T_{p 2}$ is scaled in the ordinate. $f_{0}=1 \times 10^{9} \mathrm{sec}^{-1}$. O: A-1 $\phi=0.104, \triangle$ : A-2 $\phi=0.0484, \nabla:$ A-3 $\phi=0.00792, \square$ : A-4 $\phi=0.00066, \diamond:$ A-5 $\phi=0.000049$, ○: A-6 $\phi=0.129$.

where $f_{0} \equiv 1 /\left(2 \pi \tau_{2,0}\right)$. The experimental data of samples A-1 through A-6 with respect to $T_{p 2}-\left(\ln f_{0}-\ln f\right)^{-1}$ are plotted in Fig. 10. Here the value of $f_{0}=10^{9} \mathrm{sec}^{-1}$ is adopted so the experimental data falls most suitably on a straight line. The experimental data of samples A-1 trough A-4 fall on the straight line, while samples A-5 and A-6 do not. This supports the Vogel Fulcher law's validity within the volume fraction range $0.00066 \leqslant \phi \leqslant 0.104$. Comparing $T_{p 2}$ values of $f=1000 \mathrm{~Hz}$ with the threshold dipole-dipole interaction temperature $T_{d d}$ defined by Eq. (29) in Table I, it is concluded that there is no close connection between them, which leads the Vogel-Fulcher law is not simple result of the dipoledipole interaction of two particles but cooperative effect of all the particles. The crossing points of the straight lines across $T_{p 2}$ axis in Fig. 10 give the $T_{0}$ values. Figure 11 shows the $T_{0}$ vs $\phi$ relation for the samples of A-1 through A-4. From these figures, $T_{0}$ is expressed empirically with respect to $\phi$ by

$$
T_{0} \propto \phi^{0.41}
$$

for the samples of A-1 through A-4. Zhang et al. already found that $T_{0}$ satisfies [25]

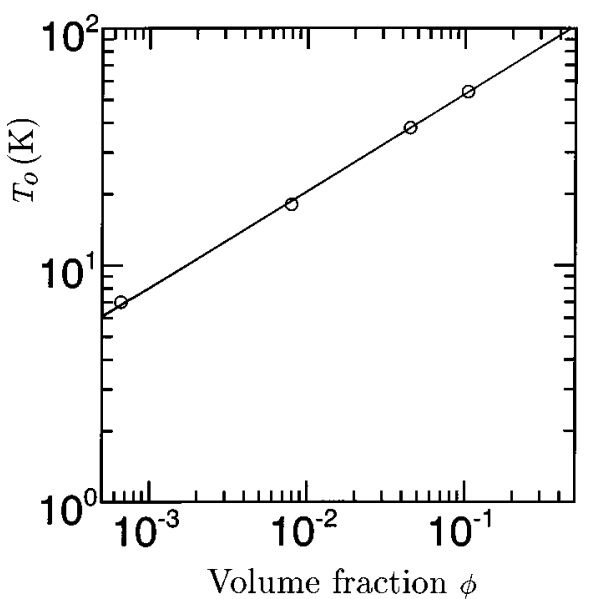

FIG. 11. $T_{0}$ vs $\phi$ relations. 


$$
T_{0} \propto \phi^{0.80}
$$

for the kerosene base magnetite particle MFs. Since exponent values of the empirical formula given by Fig. 11 remain considerably arbitrary, it is not determined whether or not the exponent differs from one MF to another or it is universal, at this stage.

Shtrikman and Wohlfarth discussed the spin glass of metal alloys diluted with ferrous atoms [46]. Introducing the mean field, they interpreted Vogel-Fulcher law. In their theory $T_{0}$ in Eq. (30) is proportional to $x^{2}$ when $x$ is small where $x$ is the atomic concentration of magnetic atoms. In our case $\phi$ corresponds to $x$. The essence of their theory is that the probability of finding a magnetic atom in the vicinity of a certain magnetic atom is proportional to $x$. If we take the clusters' generation in the MF into account, exponent values of Eqs. (32) and (33) can be obtained.

Let us denote the total volume of the clusters in the unit volume of the MF as $v_{c}$ which is naturally a function of the initial volume fraction of the colloidal particles $\phi$. Suppose the dominant term of the function $v_{c}$ is proportional to $\phi^{b}$ in a certain range value of $\phi$ then particles' volume fraction in the cluster is expressed by

$$
\frac{\phi}{v_{c}} \propto \phi^{1-b},
$$

where $b$ is a constant. Here we assumed an extreme case that all particles are in clusters and no particles are in the diluted phase. After the Shtrikman and Wohlfarth discussion, $T_{0}$ depends on $\phi$ as

$$
T_{0} \propto\left[\frac{\phi}{v_{c}}\right]^{2} \propto \phi^{2-2 b} .
$$

For example, if $b=3 / 4, T_{0}$ is proportional to $\phi^{0.5}$ and it explains the result of Fig. 11, qualitatively.

\section{Origin of magnetic aftereffect}

Up to the present, it is made clear that the $\chi^{\prime \prime}$ peak and the $\chi^{\prime \prime} / \chi^{\prime}$ peak are completely different peaks; the former is closely connected to the spin-glass-like state and the latter to the magnetic aftereffect. In Sec IV A, we partially discussed the origin of the peak of $\chi^{\prime \prime} / \chi^{\prime}$, but since the Néel relaxation is something dubious, we have to seek the real relaxation mechanism of $\chi^{\prime \prime} / \chi^{\prime}$.

The first candidate is most of the particles interact with each other but there is a small portion of particles which are completely isolated from other particles, and are subject to Néel relaxation. The fact that all the samples from A-1 through A-5 show almost the same peak temperature $T_{p 4}$ regardless of dilution, contradicts this picture because dilution should alter the noninteracting particles' distribution with respect to the particle volume $v$, and naturally $T_{p 4}$ should change with the dilution.

It is well known that bulk ferrite shows a decrease of magnetic permeability with time, which is called disaccommodation [31]. It is possible that the peak of $\chi^{\prime \prime} / \chi^{\prime}$ is due to this effect. Especially the fact that $T_{p 4}$ does not change with the dilution suggests this relaxation is a material characteris-

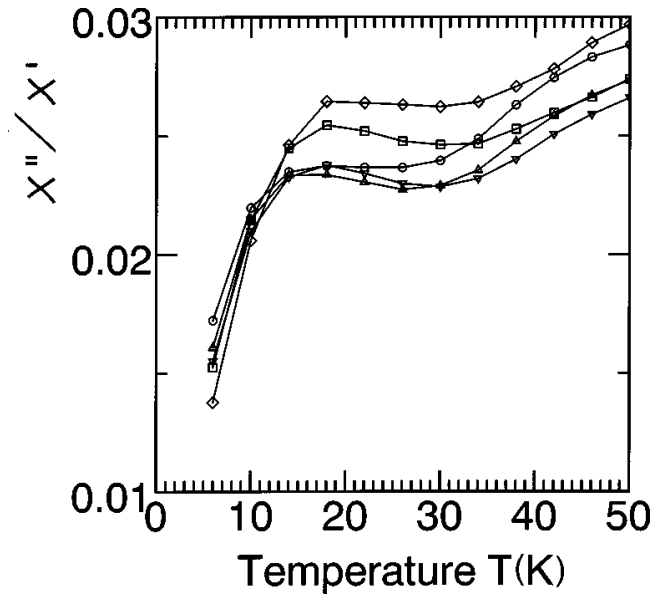

FIG. 12. $\chi^{\prime \prime} / \chi^{\prime}$ of samples $\mathrm{C}$ as a function of temperature for $f$ in the low-temperature region. $\bigcirc: 0.1 \mathrm{~Hz}, \triangle: 1 \mathrm{~Hz}, \nabla: 10 \mathrm{~Hz}, \square$ : $100 \mathrm{~Hz}, \diamond: 1000 \mathrm{~Hz}$.

tic of the colloidal particles. Two theories of disaccommodation have been proposed.

The first one is that electron hopping between $\mathrm{Fe}^{2+}$ and $\mathrm{Fe}^{3+}$ is due to the disaccommodation [47]. The activation energy of this is $0.1 \mathrm{eV}$ which coincides well with that obtained from $\chi^{\prime \prime} / \chi^{\prime}, 0.04$ to $0.1 \mathrm{eV}$ of the present experiment shown in Table I. In addition, there is no peak in MnZn ferrite particle MFs. There is no electron hopping between $\mathrm{Fe}^{2+}$ and $\mathrm{Fe}^{3+}$ in MnZn ferrite, no peak or very small one in MnZn ferrite MF is consistent with this theory.

The second one is that vacancies in the ferrite are due to the disaccommodation [48]. The activation energy in this case is $0.5 \mathrm{eV}$ which is a slightly greater than the activation energy of $\chi^{\prime \prime} / \chi^{\prime}$. Jeyadevan et al. examined the magnetite particles in the MFs and found a considerable fraction of them changes from magnetite to $\gamma-\mathrm{Fe}_{2} \mathrm{O}_{3}$ [49]. Here the octahedral site in magnetite is vacant. If this fact is a general characteristic of ultrafine ferrite, the vacancy theory is still a candidate to explain the $\chi^{\prime \prime} / \chi^{\prime}$ peaks.

Finally, we mention the small but peculiar shoulder or peak of $\chi^{\prime \prime} / \chi^{\prime}$ as a function of temperature at $18 \mathrm{~K}$ for sample $\mathrm{C}$ or MnZn Ferrite particles MF. As is shown in Fig. 12 , this shoulder does not show a temperature shift with the frequency $f$, which means this relaxation process is not the thermal activation type. Tejada et al. measured the magnetic viscosity $S_{v}$ of MFs as a function of temperature under $10 \mathrm{~K}$ [50]. They found that there remains a residual in $S_{v}$, even as $T$ decreases to 0 . They attributed it to the quantum tunneling effect of dipoles. Since the present shoulder is not the thermal activation type, there is a possibility of a quantum tunneling effect for this shoulder. But at this stage, the origin of this shoulder is also open to question.

\section{CONCLUSION}

We measured the complex magnetic susceptibility $\chi$ of the MF (magnetic fluid) as a function of temperature in a weak ac field of 1 Oe amplitude from 0.1 to $1000 \mathrm{~Hz}$ by a SQUID magnetometer. It is clarified from the present experiment that the temperature dependence of $\chi$ is due to mainly two effects: one is the magnetic aftereffect and the other one is the generation of spin-glass-like state when some kinds of 
MFs are frozen. The former phenomenon appears when we plot the ratio of the imaginary and the real part $\chi^{\prime \prime} / \chi^{\prime}$ as a function of temperature. There appears a peak in the vicinity of $30 \mathrm{~K}$ and the peak temperature dependence on ac field frequency is of Arrhenius or thermal activation type except for a small shoulder for MnZn ferrite particle MFs. The origin of the magnetic after effect is not identified at present stage, but the Néel relaxation of noninteracting particles, electron-electron hopping between $\mathrm{Fe}^{2+}$ and $\mathrm{Fe}^{3+}$ in the magnetite particles, and the vacancies in $\gamma-\mathrm{Fe}_{2} \mathrm{O}_{3}$ particles are a possible cause of this magnetic aftereffect. The quantum tunneling effect is a possible candidate of the nonthermal activation-type relaxation in MnZn ferrite particle MFs.

When a MF is cooled down a phase separation occurs in the MF and small droplets of dense phase or clusters and diluted phase are generated. In the clusters the number density of the colloidal particles increases dramatically, and it leads the dramatic increase in the dipole-dipole interaction among the particles in the cluster. If this dipole-dipole interaction strength gets strong enough, the dipoles in the cluster order in the same direction and achieve a ferromagneticlike state. The magnetic susceptibility $\chi$ as a function of temperature does not show any peaks in the frozen state of MFs for a few samples in the present experiment. It corresponds to the strong interaction strength. On the other hand, if the interaction reaches intermediate strength, the dipoles form a spin-glass-like state, which leads to the appearance of a cusp in the magnetic susceptibility of the MF as a function of temperature in the frozen state and the peak temperature dependence on the relaxation time obeys the Vogel-Fulcher law for the MF of the particles volume fraction $\phi$ satisfying $0.0007 \leqslant \phi \leqslant 0.104$. The parameter $T_{0}$ of temperature dimension in Eq. (30), which corresponds to the dipole-dipole interaction strength, is empirically proportional to $\phi^{0.41}$. If we take the cluster generation into account, this exponent is derived from theory of Shtrikman and Wohlfarth [46].

\section{ACKNOWLEDGMENTS}

The author expresses his thanks to Dr. W. Luo for critical discussions on the present study and J. Zhang for helping perform the experiment. The author also acknowledges them for showing him their experimental results of the kerosene base magnetite particle MF before publication [25]. The MFs were provided by Matsumoto Yushi-Seiyaku Co. Ltd. This work was partly supported by NSF DMR and the NSF (W. Luo).

\section{APPENDIX A}

The dynamics of magnetic spins are usually predicted by the Landau-Lifshitz equation [30]. Actually, Raikher and Stepanov derived their theory of ferromagnetic resonance of MFs from the Landau-Lifshitz equation [51]. The magnetic dipole of the colloidal particle in MFs is, however, the composite of the spins of all the ferromagnetic or ferrimagnetic atoms in the colloidal particle and the relaxation frequency decreases by less than $1000 \mathrm{~Hz}$ in the low temperature. It leads to the dipole's direction being almost localized in the two opposite directions of minimum energy. Therefore it is more suitable to adopt the following two-state model.

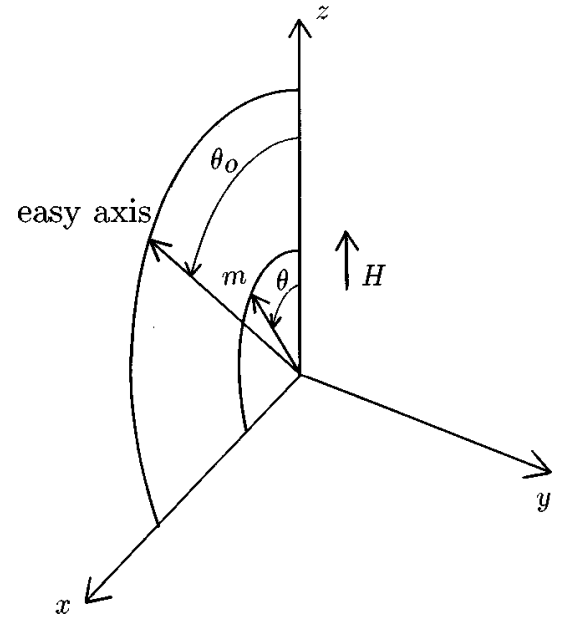

FIG. 13. Configuration of Cartesian coordinate system $(x, y, z)$ and polar coordinate system $(r, \theta, \varphi)$ with respect to the easy axis, the dipole $\boldsymbol{m}$, and the external field $\boldsymbol{H}$.

Let us consider a colloidal particle of volume $v$ with magnetic dipole $\boldsymbol{m}$ having an uniaxial magnetic property. As the MF is zero field cooled, the direction of the easy axis in each particle is distributed randomly. Let us assume that the dipole $\boldsymbol{m}$ is almost fixed in the easy axis of the particles. Therefore the problem is reduced to a two-state model problem, i.e., the dipoles are fixed in one direction of the easy axis or in the opposite direction. The probability of directing to the two direction is the same when there is no external field.

We introduce a Cartesian and polar coordinate system as shown in Fig. 13. Now we look at one particle. Let the easy axis of it lie in the $x z$ plane without breaking the generality. Let the angle between this axis and the $z$ axis be $\theta_{0}$ and let the external field $\boldsymbol{H}$ be applied in the $z$ direction. Let $\boldsymbol{m}$ be in the $x z$ plane and make an angle $\theta$ with the $z$ axis.

\section{Uniaxial easy axis model}

The magnetic energy $E$ is expressed by

$$
E=-K v \cos ^{2}\left(\theta-\theta_{0}\right)-m H \cos \theta
$$

where $K$ is the anisotropy constant. When $\boldsymbol{m}$ gets off the $x z$ plane, $E$ increases. Therefore we need not consider the case when $\boldsymbol{m}$ is off the $x z$ plane. We consider the weak external field case, i.e.,

$$
\frac{m H}{K v} \ll 1 .
$$

The presence of the external field, $\boldsymbol{H}$ changes the energy minimum and maximum positions of $\boldsymbol{m}$ direction slightly. Let the angle $\theta$ of the new minimum position be $\theta=\theta_{0}+t_{1}$. $\left(t_{1} \ll 1\right)$. Then $E$ is expressed by

$$
E=-K v\left[1-\frac{t_{1}^{2}}{2}-\frac{m H}{K v} \sin \theta_{0} t_{1}+\frac{m H}{K v} \cos \theta_{0}\right] .
$$




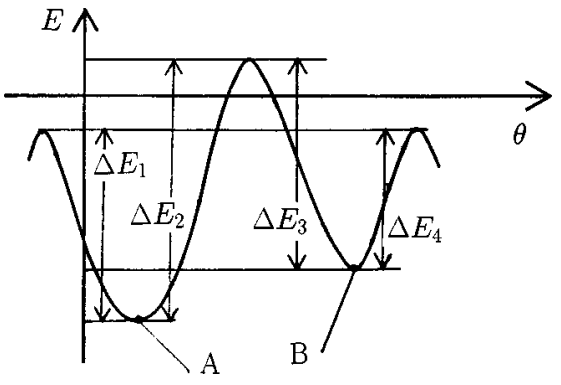

FIG. 14. Schematic figure of energy minima and maxima with respect to the angle $\theta$ between the external field $\boldsymbol{H}$ and the dipole $m$.

$$
\begin{gathered}
\frac{\partial E}{\partial t_{1}}=m H \sin \theta_{0}+2 K v t_{1}=0, \\
t_{1}=-\frac{1}{2} \frac{m H}{K v} \sin \theta_{0},
\end{gathered}
$$

the minimum energy value $E_{\min 1}$,

$$
E_{\min 1}=-K v\left[1+\frac{m H}{K v} \cos \theta_{0}\right],
$$

is obtained. Here we neglected the higher power terms of $m \mathrm{H} / \mathrm{Kv}$.

In the same way, the maximum energy value $E_{\max 1}$ in the vicinity of $\theta=\theta_{0}+\pi / 2$, another energy minimum $E_{\min 2}$ in the vicinity of $\theta=\theta_{0}+\pi$, and another energy maximum $E_{\max 2}$ in the vicinity of $\theta=\theta_{0}+3 \pi / 2$, are expressed, respectively, by

$$
\begin{gathered}
E_{\max 1}=K v\left[\frac{m H}{K v} \sin \theta_{0}\right] \quad \text { at } \theta=\theta_{0}+\frac{\pi}{2}+\frac{1}{2} \frac{m H}{K v} \cos \theta_{0} \\
E_{\min 2}=K v\left[-1+\frac{m H}{K v} \cos \theta_{0}\right] \\
\text { at } \theta=\theta_{0}+\pi+\frac{1}{2} \frac{m H}{K v} \sin \theta_{0} \\
E_{\max 2}=-K v\left[\frac{m H}{K v} \sin \theta_{0}\right] \\
\text { at } \theta=\theta_{0}+\frac{3 \pi}{2}-\frac{1}{2} \frac{m H}{K v} \cos \theta_{0} .
\end{gathered}
$$

Figure 14 shows schematically the energy $E$ vs the angle $\theta$.

\section{General anisotropy potential}

In the previous section we assumed the anisotropy energy as $-K v \cos ^{2}\left(\theta-\theta_{0}\right)$, but as shown in the derivation process of the minimum and the maximum energy positions, a more general form of the anisotropy potential is possible. We denote it as $V\left(\theta-\theta_{0}\right)$. If $V(x)$ satisfies the conditions (1) $V(x)$ is a fourfold, mirror symmetric and periodic function of $2 \pi$ with respect to $x$. (2) $V(x)$ has a minimum and a maximum at $x=0$ and $x=\pi / 2$, respectively, and in the vicinity of $x=0$, and $x=\pi / 2, V$ can be expressed by

$$
\begin{gathered}
V(x) \cong V_{0}+\frac{1}{2} V_{1} x^{2}, \\
V(x) \cong V_{0}^{\prime}-\frac{1}{2} V_{1}^{\prime}\left[x-\frac{\pi}{2}\right]^{2},
\end{gathered}
$$

where $V_{0}$ and $V_{0}^{\prime}$ are constants, and $V_{1}$ and $V_{1}^{\prime}$ are positive constants, respectively, the same conclusions as in the previous section are obtained. Therefore the anisotropy energy is not only due to the shape anisotropy and the magnetocrystalline anisotropy energy but also to other potential energies such as electron hopping if it satisfies the above conditions.

\section{Derivation of Eq. (1)}

The left-hand and right-hand side barrier heights $\Delta E_{1}$ and $\Delta E_{2}$ at the lowest energy point $\mathrm{A}$ in Fig. 14 are expressed by

$$
\begin{aligned}
& \Delta E_{1}=K v-m H \sin \theta_{0}+m H \cos \theta_{0}, \\
& \Delta E_{2}=K v+m H \sin \theta_{0}+m H \cos \theta_{0} .
\end{aligned}
$$

In the same way, the left-hand and right-hand side barrier heights $\Delta E_{3}$ and $\Delta E_{4}$ at the next lowest energy point $\mathrm{B}$ in Fig. 14 are expressed by

$$
\begin{aligned}
& \Delta E_{3}=K v+m H \sin \theta_{0}-m H \cos \theta_{0}, \\
& \Delta E_{4}=K v-m H \sin \theta_{0}-m H \cos \theta_{0} .
\end{aligned}
$$

Let the probabilities of the dipole $\boldsymbol{m}$ existing at energy minimum points $\mathrm{A}$ and $\mathrm{B}$ in Fig. 14 as $p_{+}(v)$ and $p_{-}(v)$, respectively. Here we denote $p_{ \pm}(v)$ because we will take the particles volume distribution into account afterwards. Then the rate equation is expressed by

$$
\begin{aligned}
\frac{d p_{+}(v)}{d t}= & -c\left\{p_{+}(v)\left[\exp \left(-\frac{\Delta E_{1}}{k_{B} T}\right)+\exp \left(-\frac{\Delta E_{2}}{k_{B} T}\right)\right]\right. \\
& \left.-p_{-}(v)\left[\exp \left(-\frac{\Delta E_{3}}{k_{B} T}\right)+\exp \left(-\frac{\Delta E_{4}}{k_{B} T}\right)\right]\right\},
\end{aligned}
$$

where $c$ is a constant. Inserting Eqs. (A12)-(A15) into Eq. (A16) and expanding in powers of $m H / k_{B} T$, we obtain

$$
\begin{aligned}
\frac{d p_{+}(v)}{d t}= & -c\left\{2 p_{+}(v)\left(1-\frac{m H \cos \theta_{0}}{k_{B} T}\right) \exp \left[-\frac{K v}{k_{B} T}\right]\right. \\
& \left.-2 p_{-}(v)\left(1+\frac{m H \cos \theta_{0}}{k_{B} T}\right) \exp \left[-\frac{K v}{k_{B} T}\right]\right\} \\
= & -2 c\left[\left[p_{+}(v)-p_{-}(v)\right]-\frac{m H \cos \theta_{0}}{k_{B} T}\right] \\
& \times \exp \left[-\frac{K v}{k_{B} T}\right]
\end{aligned}
$$

Here we neglected the terms of the higher powers of $m H / k_{B} T$ than the first and the relation 


$$
p_{+}(v)+p_{-}(v)=1
$$

was used.

Taking the particles' volume distribution into account, the magnetization $M$ is expressed by

$$
\begin{aligned}
M= & N \int_{0}^{\infty} d v f_{\text {dis }}(v) \int \frac{d \Omega}{2 \pi} m(v)\left[p_{+}(v) \cos \left(\theta_{0}+t_{1}\right)\right. \\
& \left.+p_{-}(v) \cos \left(\theta_{0}+\pi+t_{3}\right)\right] \\
\approx & N \int_{0}^{\infty} d v f_{\text {dis }}(v)\left\{\frac{m^{2} H}{3 K v}+m \int \frac{d \Omega}{2 \pi}\right. \\
& \left.\times\left[p_{+}(v)-p_{-}(v)\right] \cos \theta_{0}\right\},
\end{aligned}
$$

where $N$ is the number density of the particles, $m(v)$ is expressed by

$$
m(v)=M_{S} v
$$

$d \Omega$ is the differential steric angle, and the integration is done over the upper hemisphere. Finally $M$ is expressed by

$$
\begin{aligned}
M-\chi_{S} H= & N M_{S} \int_{0}^{\infty} d v v f_{\mathrm{dis}}(v) \int \frac{d \Omega}{2 \pi} \\
& \times\left[p_{+}(v)-p_{-}(v)\right] \cos \theta_{0} .
\end{aligned}
$$

Here $\chi_{S}$ is the adiabatic susceptibility defined by

$$
\chi_{S} \equiv \frac{M_{S}^{2} \phi}{3 K}
$$

Using Eqs. (A17) and (A21)

$$
\begin{aligned}
\frac{d}{d t}\left(M-\chi_{S} H\right)= & N M_{S} \int_{0}^{\infty} d v v f_{\mathrm{dis}}(v) e^{-K v / k_{B} T} \\
& \times \int \frac{d \Omega}{2 \pi}\left[p_{+}(v)-p_{-}(v)\right] \cos \theta_{0} \\
& +\frac{4 c M_{S}^{2} N H}{3 k_{B} T} \int_{0}^{\infty} d v v^{2} f_{\mathrm{dis}}(v) e^{-K v / k_{B} T},
\end{aligned}
$$

is obtained. Now we adopt proper $v_{1}$ and $v_{2}$ which are in the vicinity of $v_{0}$ in Eq. (10) and satisfy the equations

$$
\begin{gathered}
\int_{0}^{\infty} d v v f_{\mathrm{dis}}(v) e^{-K v / k_{B} T} \int \frac{\mathrm{d} \Omega}{2 \pi}\left[p_{+}(v)-p_{-}(v)\right] \cos \theta_{0} \\
=e^{-K v_{1} / k_{B} T} \int_{0}^{\infty} d v v f_{\mathrm{dis}}(v) \int \frac{d \Omega}{2 \pi} \\
\quad \times\left[p_{+}(v)-p_{-}(v)\right] \cos \theta_{0}
\end{gathered}
$$

$$
\int_{0}^{\infty} d v v^{2} f_{\mathrm{dis}}(v) e^{-K v / k_{B} T}=e^{-} K v_{2} / k_{B} T \int_{0}^{\infty} d v v^{2} f_{\mathrm{dis}}(v)
$$

Then Eq. (A23) is transformed to

$$
\begin{aligned}
\frac{d}{d t}\left(M-\chi_{S} H\right)= & -4 c e^{-K v_{1} / k_{B} T}\left(M-\chi_{S} H\right) \\
& +\frac{4 c M_{S}^{2} N}{3 k_{B} T} e^{-K v_{2} / k_{B} T} \bar{v}^{-} H,
\end{aligned}
$$

where $\vec{v}^{2}$ is defined by

$$
\vec{v}^{2}=\int_{0}^{\infty} d v v^{2} f_{\text {dis }}(v)
$$

Finally assuming

$$
v_{1}=v_{2}=v^{*}
$$

where $v^{*}$ is approximately the same as $v_{0}$ in Eq. (10), and defining $\tau_{4}$ and the isothermal susceptibility $\chi_{T}$ as

$$
\tau_{4} \equiv \frac{1}{4 c} e^{K v^{*} / k_{B} T}
$$

$$
\chi_{T} \equiv \frac{M_{S}^{2} N \vec{v}^{2}}{3 k_{B} T}
$$

The equation

$$
\frac{d}{d t}\left(M-\chi_{S} H\right)=-\frac{1}{\tau_{4}}\left[M-\chi_{S}\left(1+\frac{\chi_{T}}{\chi_{S}}\right) H\right]
$$

is obtained, and is equivalent to Eq. (1).

\section{APPENDIX B}

Let us assume that the colloidal particle is a sphere of radius $r$. Then the volume $v$ is expressed by

$$
v=\frac{4 \pi r^{3}}{3}
$$

Next let us denote the log-normal distribution function with respect to the radius $r$ as $\bar{f}_{\text {dis }}(r)$ which is expressed by

$$
\bar{f}_{\text {dis }}(r)=\frac{1}{\sqrt{2 \pi} \sigma_{1} r} \exp \left[\frac{-\left[\ln \left(r / r_{1}\right)\right]^{2}}{2 \sigma_{1}^{2}}\right]
$$

By definition, 


$$
\bar{f}_{\text {dis }}(r) d r=f_{\text {dis }}(v) d v,
$$

holds. From Eqs. (10) and (B1)-(B3),

$$
v_{0}=\frac{4 \pi r_{1}^{3}}{3}
$$

and

$$
\sigma=3 \sigma_{1}
$$

are derived. In the present case, since $r_{1}=3.35 \mathrm{~nm}$ and

[1] For textbooks of MF(s), refer to Refs. [2] and [3].

[2] R. E. Rosensweig, Ferrohydrodynamics (Cambridge University Press, Cambridge, 1985).

[3] S. Taketomi and S. Chikazumi, Magnetic Fluids-Principle and Application (Nikkan Kogyo Shinbun Tokyo, 1988); S. Taketomi and S. Chickazuml, ibid. (Mir, Moscow, 1993).

[4] L. Néel, Ann. Geophys 5, 99 (1949).

[5] Refer to Refs. [2] and [3].

[6] W. Luo, S. R. Nagel, T. F. Rosenbaum, and R. E. Rosensweig, Phys. Rev. Lett. 67, 2721 (1991).

[7] H. D. Williams, K. O'Grady, S. W. Charles, and K. J. Davies, J. Magn. Magn. Mater. 122, 134 (1993).

[8] S. Taketomi, M. Ukita, M. Mizukami, H. Miyajima, and S. Chikazumi, J. Phys. Soc. Jpn. 56, 3362 (1987).

[9] A. O. Tsebers(Cebers), Magni. Gidrodin. 18, 42 (1982) [Magnetohydrodynamics 18, 137 (1982)].

[10] K. Sano and M. Doi, J. Phys. Soc. Jpn. 52, 2810 (1983).

[11] K. I. Morozov, A. F. Pshenichnikov, Yu. L. Raikher, and M. I. Shliomis, J. Magn. Magn. Mater. 65, 269 (1987).

[12] M. A. Martsenyuk, Yu. L. Raikher, and M. I. Shliomis, Zh. Eksp. Teor. Fiz. 65, 834 (1973) [Sov. Phys. JETP 38, 413 (1974).]

[13] E. P. Wohlfarth, J. Magn. Magn. Mater. 39, 39 (1983).

[14] R. W. Chantrell and E. P. Wohlfarth, J. Magn. Magn. Mater. 40, 1 (1983).

[15] B. K. P. Scaife, J. Phys. D 19, L195 (1986).

[16] P. C. Fannin, B. K. P. Scaife, and S. W. Charles, J. Phys. E 19, 238 (1986).

[17] W. F. Brown, Phys. Rev. 130, 1677 (1963).

[18] P. Debye, Polar Molecules (Chemical Catalog Co. New York, 1929).

[19] P. C. Fannin, S. W. Charles, and T. Relihan, J. Magn. Magn. Mater. 149, 29 (1995).

[20] M. Hanson and C. Johansson, J. Magn. Magn. Mater. 101, 45 (1991).

[21] A. Tari, J. Popplewell, S. W. Charles, D. St. P. Bunbury, and K. M. Alves, J. Appl. Phys. 54, 3351 (1983).

[22] A. A. Minakov, I. V. Zaitsev, and U. I. Lesnih, J. Magn. Magn. Mater. 85, 60 (1990).

[23] I. Abu-Aljarayesh, A. Al-Rawi, and H. Abu-Safia, J. Magn. Magn. Mater. 119, 87 (1993).

[24] T. Jonsson, J. Mattsson, C. Djurberg, F. A. Khan, P. Nordblad, and P. Svedlindh, Phys. Rev. Lett. 75, 4138 (1995).

[25] J. Zhang, C. Boyd, and W. Luo, Phys. Rev. Lett. 77, 390 (1996). $\sigma_{1}=0.45$ from Ref. [34], $v_{0}=157 \mathrm{~nm}^{3}$ and $\sigma=1.35$ are obtained from Eqs. (B4) and (B5). Using the formulas

$$
\begin{aligned}
\vec{v}^{n} & \equiv \int_{0}^{\infty} v^{n} f_{\text {dis }}(v) d v \\
& =v_{0}^{n} \exp \left[\frac{n^{2} \sigma^{2}}{2}\right]
\end{aligned}
$$

Eq. (11) is also obtained.

[26] S. Taketomi, N. Inaba, H. Takahashi, and H. Miyajima, J. Phys. Soc. Jpn. 59, 3077 (1990).

[27] H. Miyajima, N. Inaba, S. Taketomi, M. Sakurai, and S. Chikazumi, J. Appl. Phys. 63, 4267 (1988).

[28] H. Miyajima, N. Inaba, S. Taketomi, and S. Chikazumi, J. Phys. (Paris) 49, C8-1843 (1988).

[29] S. Taketomi, H. Takahashi, N. Inaba, H. Miyajima, and S. Chikazumi J. Phys. Soc. Jpn. 59, 2500 (1990).

[30] Though the magneto-optical effect of the magnetic fluid of A-7 was different from that of A-1 (see Ref. [26]), it was revealed in the present experiment that the $\chi$ 's of them are the same. Therefore $\chi$ of A-7 is not shown.

[31] S. Chikazumi, Physics of Magnetism (Wiley, New York, 1964).

[32] K. Abe, Y. Miyamoto, and S. Chikazumi, J. Phys. Soc. Jpn. 41, 1894 (1976).

[33] R. W. Chantrell, J. Popplewell, and S. W. Charles, IEEE Trans. Magn. MAG-14, 975 (1978).

[34] S. Taketomi, H. Takahashi, N. Inaba, and H. Miyajima, J. Phys. Soc. Jpn. 60, 3426 (1991). In this reference, a distribution function $\bar{f}_{\text {dis }}(r)$ with respect to the radius $r$ was used. The relation between these two distribution functions is shown in Appendix B.

[35] J. Frenkel, The Kinetic Theory of Liquids (Dover, New York, 1955).

[36] Refer to Chap. 1.3 of Ref. [3].

[37] L. Lundgren, P. Svedlindh, and O. Beckman, J. Magn. Magn. Mater. 25, 33 (1981).

[38] C. Kittel, Introduction to Solid State Physics, 3rd ed. (Wiley, New York, 1953), Chap. 14.

[39] S. Taketomi, H. Takahashi, N. Inaba, and H. Miyajima, J. Phys. Soc. Jpn. 60, 1689 (1991).

[40] H. Wang, Y. Zhu, C. Boyd, W. Luo, A. Cebers, and R. E. Rosensweig, Phys. Rev. Lett. 72, 1929 (1994).

[41] J. Zhang and W. Luo (unpublished).

[42] M. Shimada, Jisei Ryuutai Rengou Kouenkai Ronbun shuu [Proc. Jpn. Magn. Fluid Research (in Japanese)] 40 (1991).

[43] M. Doi and H. T. See, J. Phys. Soc. Jpn. 61, 2090 (1992).

[44] For a review of spin glasses, see K. Bindr and A. P. Young, Rev. Mod. Phys. 58, 801 (1986).

[45] H. Mamiya and I. Nakatani, J. Magn. Soc. Jpn. 20, 293 (1996).

[46] S. Shtrikman and E. P. Wohlfarth, Phys. Lett. 85A, 467 (1981).

[47] J. L. Snoek, New Developments in Ferromagnetic Mmaterials (Elsevier, Amsterdam, 1949). Sec. 16. 
[48] K. Ohta, J. Phys. Soc. Jpn. 16, 250 (1961).

[49] B. Jeyadevan, K. Tohjo, and K. Nakatsuka, Funtai oyobi Funmatsu Yakin [J. Powder Powder Metallurgy (in Japanese)] 41, 113 (1994).
[50] J. Tejada, Ll. Balcells, S. Lindroth, R. Perzynski, B. Rigau, B. Barbara, and J. C. Bacri, J. Appl. Phys. 73, 6952 (1993).

[51] Yu. L. Raikher and V. I. Stepanov, J. Magn. Magn. Mater. 149, 34 (1995). 